\title{
ASPECTOS VALORIZADOS POR PORFISSIONAIS DE ENFERMAGEM NA HIGIENE PESSOAL E NA HIGIENE CORPORAL DO PACIENTE*
}

Elóide André Oliveira** Telma Ribeiro Garcia*** Lenilde Duarte de Sá ${ }^{\star \star \star \star}$

\section{Resumo}

Survey descritivo, com o qual se objetivou identificar que aspectos da higiene corporal são valorizados pelos componentes da equipe de enfermagem, na higiene corporal pessoal e na higiene corporal do paciente. A pesquisa foi desenvolvida em oito instituições públicas de saúde e a amostra foi composta por 126 profissionais de enfermagem, correspondendo a $12,8 \%$ do total de 986 componentes das equipes de enfermagem destas instituições. Na higiene corporal pessoal, chamou a atenção o fato de que os cuidados com as unhas e com os cabelos tenham suplantado, no número de citações dos profissionais de enfermagem, aspectos básicos como o cuidado com as mãos. Na higiene corporal do paciente, o banho foi o aspecto considerado mais importante pelas três categorias profissionais; a higiene oral, pelas(os) enfermeiras(os) e auxiliares de enfermagem; e as unhas, pelas(os) enfermeiras(os) e técnicas(os) de enfermagem. Descritores: saúde pública, higiene, hogiene corporal, assistência de enfermagem.

\begin{abstract}
Descriptive survey aimed at identifying which aspects of body hygiene are valued by a nursing staff both in regard to personal body hygiene and to patients body hygiene. This survey has been conducted inside eight public health institutions, and the sample consisted of 126 nursing professionals, corresponding to $12.8 \%$ of a total of 986 people of the nursing personnel in such institutions. When considering personal body hygiene, the number of nail and hair care related quotes was higher than the number of answers concerning basic aspects such as hands hygiene, and this fact has called our attention. In patients body hygiene, a shower was the highest regarded aspect by the nursing staff; oral hygiene by registered nurses and nursing aids; and nail care by registered nurses and nursing technicians.

Descriptors: Public health; Hygiene; Body hygiene; nursing care

Title: Aspects valued by Nursing professionals in regard to personal body' hygiene and patients body hygiene
\end{abstract}

\section{Resumen}

Estudio descriptivo, cuyo objetivo fue identificar qué aspectos de aseo corporal son valorados por los componentes de Enfermería, en el aseo personal y en el aseo corporal de los pacientes. La investigación fue desarrollada en ocho instituciones públicas, y la muestra estaba compuesta por 126 profesionales de enfermería, correspondiendo al $12,8 \%$ de un total de 986 integrantes de los equipos de Enfermería de dichas instituciones. En la higiene corporal personal, llamó la atención el hecho de que el aseo de las unas y del pelo hayan suplantado el de cuidado con las manos, en los aspectos citados por los profesionales. En la higiene corporal del paciente, el baño fue el aspecto que se consideró como el más importante dentro de las tres categorías profesionales. Otros aspectos, así fueron considerados relevantes:

la higiene bucal, por las enfermeras y los auxiliares de enfermería; y las unas, por enfermeras $y$ técnicos de enfermería. Descriptores: Salud pública; higiene; higiene corporal; asistencia de enfermería

Título: Los aspectos que los profesionales de enfermería más valoran en la higiene corporal personal y en la higiene corporal dei paciente
1 Introdução

O vocábulo higiene "deriva da deusa Hygiéia, reverenciada em Atenas como protetora da saúde, do bem estar orgânico"(1:IX). O termo admite vários adjuntos adnominais que o complementam e expandem seu significado: higiene coletiva, higiene mental, higiene profissional, higiene pública, higiene social, higiene da habitação, higiene da alimentação e higiene corporal, entre outros. Este último conceito - higiene corporal - é o alvo de nossa atenção neste trabalho.

Num esforço de resgatar e situar a história dos hábitos de higiene corporal, Vigarello(2) narrou os caminhos e as concepções reveladores da transformação do conceito e, em especial, das práticas de higiene, o que envolveu os órgãos dos sentidos, como o olfato e a visão, que foram se aguçando e se refinando em relação à imagem e às sensações corporais. Segundo sua narrativa, a higiene corporal foi sendo, gradualmente, deslocada do espaço público para o privado, tendo como suporte as regras culturais e o conhecimento científico específicos de cada época. $O$ autor considera que, no início do século XIX, o vocábulo higiene ocupa um lugar inédito pois, a partir de então, não é mais adjetivo que qualifica a saúde, mas "o conjunto de dispositivos e saberes que favorecem a sua manutenção. É uma disciplina específica dentro da medicina. É um corpo de conhecimento, não mais um qualitativo físico. Com esse título subitamente um campo se especializou"(2:186).

A especialização adquirida pela higiene aparece num contexto em que a química, a fisiologia e a história natural se desenvolvem cientificamente. Nesse sentido, considera ser impossível evocar a higiene sem fazer dela um ramo específico do conhecimento médico.

Ainda de acordo com o autor citado, a história da limpeza corporal é, também, uma história social. Assumindo-se esse ponto de vista, há que se considerar a contemporaneidade da instauração da Higiene como campo de saber específico e do nascimento da Enfermagem Moderna. Do ponto de vista social, tanto a Higiene quanto a Enfermagem estão, à época, envolvidos por concepções advindas da cientificidade, do contexto político e cultural específicos do século XIX.

O nascimento da Enfermagem Moderna é associado à figura de Florence Nightingale (1820-1910), que parece ter sido influenciada, entre outros aspectos, pelas discussões científicas sobre a água e o seu importante papel na salubridade dos

* Higiene corporal na visão da equipe de enfermagem: estudo em instituições públicas de saúde do município de João Pessoa-PB. (Dissertação de Mestrado). João Pessoa: Programa de Pós-Graduação em Enfermagem, Universidade da Paraíba; $2002.83 f$

** Enfermeira. Mestre em Enfermagem de Saúde Pública pela UFPB. Coordenadora Técnica e Docente da Escola Técnica de Enfermagem Materdei, João Pessoa - PB .

*** Enfermeira. Doutora em Enfermagem pela EERP-USP. Profa. Adj. IV do Departamento de Enfermagem de Saúde Pública e Psiquiatria, CCS/UFPB.

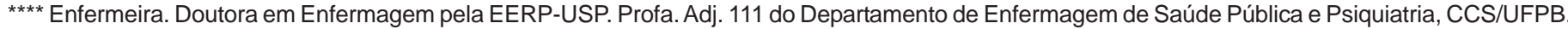
E-mail do autor:tegarcia@ccs.ufpb.br 
ambientes, principalmente das cidades modernas. No século XIX, ocorreram algumas inovações na prática da higiene, uma das quais foi o uso do sabão no banho. Água morna e sabão, para a enfermeira inglesa, era uma combinação que, verdadeiramente, promovia limpeza. No que concerne à higiene corporal, Florence Nightingale insistia sobre o cuidado com a pele, vista por ela como depuradora de secreções nocivas à saúde e necessitando, portanto, de limpeza por meio do "banho com água tépida, sabão e esfregaços"(3:108). O objetivo de tal prática não era somente de limpeza, mas de alívio e conforto, o que colocaria o indivíduo em condições adequadas para o seu restabelecimento físico ou para a manutenção de sua saúde. Do ponto de vista das exercentes da Enfermagem, acreditava não ser necessário "dizer a uma enfermeira que deve estar sempre limpa [ ... ], uma vez que a maior parte da enfermagem consiste em preservar a limpeza"(3:100). Além disso, recomendava: "as enfermeiras devem ter o cuidado de lavar freqüentemente as mãos durante o dia. Se lavarem também o rosto, tanto melhor"(3:107).

Higiene e, de modo especial, higiene corporal, têm sido conceitos valorizados pela Enfermagem ao longo do tempo. A literatura reforça a importância do conceito para a profissão, incluindo-se no rol de atribuições de seus exercentes a de desenvolver nos pacientes hábitos de higiene corporal adequados. A Enfermagem apóia-se em fundamentos científicos para desenvolver sua prática profissional. Acredita se portanto que, ao se realizar um procedimento que envolve a higiene corporal, quer seja a própria ou a do paciente, estejam sendo aplicados conhecimentos acumulados ao longo da história da profissão e dos campos afins.

Hábitos, costumes e determinantes culturais são valorizados como fatores importantes nesse processo e, no cotidiano das instituições de saúde, temos observado uma certa desarticulação entre os conhecimentos transmitidos durante o período de formação profissional e os comportamentos esperados na prática dos componentes da equipe de enfermagem quanto a cuidados higiênicos.

Dessa forma, este estudo objetivou verificar como os componentes da equipe de enfermagem definem os conceitos de higiene e higiene corporal; e identificar que aspectos da higiene corporal são valorizados pelos componentes da equipe de enfermagem, tanto no que diz respeito à higiene corporal pessoal como à higiene corporal do paciente sob seus cuidados profissionais.

\section{Material e método}

Realizou-se um estudo descritivo, do tipo survey, com abordagem quanti-qualitativa de análise de dados, que envolveu, como população de referência, profissionais de enfermagem que trabalhavam em ambientes de internação hospitalar. O estudo foi desenvolvido em instituições públicas de saúde, prestadoras de serviços de internação hospitalar, do município de João Pessoa-PB. Para a definição da população e da amostra do estudo, foram percorridos os passos descritos a seguir.

Para estabelecer o número de instituições que seriam incluídas na pesquisa, obteve-se, através do Conselho Regional de Enfermagem da Paraíba (COREN-PB), a relação de instituições públicas prestadoras de serviços de internação hospitalar do município de João Pessoa-PB. Realizou-se um sorteio aleatório de nove das 17 instituições constantes na relação obtida, buscando-se com isso garantir um percentual mínimo de $50 \%$ de instituições incluídas na pesquisa. A seguir, entrou-se em contato com a Direção Geral e com a Gerência de Enfermagem das instituições sorteadas. explicando-Ihes os objetivos pretendidos e a metodologia a ser utilizada, com vistas à obtenção do consentimento para a execução da pesquisa e para o acesso aos componentes da equipe de enfermagem dessas instituições, nos três turnos de serviço (manhã, tarde e noite), para distribuição e recolhimento dos instrumentos de coleta de dados. Garantidas essas condições, o projeto foi encaminhado à apreciação do Comitê de Ética em Pesquisa do Centro de Ciências da Saúde - CCS/UFPB.

No decorrer da pesquisa, tomou-se a decisão de excluir uma das instituições sorteadas, por haver sido observado pouco interesse dos componentes da equipe de enfermagem em responder e devolver o instrumento de coleta de dados distribuído dentro do prazo estabelecido, a despeito dos esforços despendidos. O número de instituições inseridas na pesquisa ficou, portanto, reduzida a oito, correspondendo a $47,1 \%$ do total de 17 instituições com possibilidade de inclusão.

A população do estudo foi constituída por todos os profissionais de enfermagem que trabalhavam nessas oito instituições. Foram considerados os aspectos éticos da pesquisa envolvendo seres humanos, em conformidade com o previsto na Resolução no 196/96 do Conselho Nacional de Saúde(4), principalmente no que diz respeito à obtenção do consentimento livre e esclarecido e à garantia do anonimato dos participantes durante a divulgação, escrita ou oral, das informações colhidas na pesquisa. Para inclusão na amostra do estudo, além da participação voluntária, foram estabelecidos os seguintes critérios: ser enfermeiro, técnico ou auxiliar de enfermagem; trabalhar em unidades de internação das instituições selecionadas para o estudo; e devolver o instrumento de coleta de dados dentro do prazo previamente estabelecido.

Para a coleta dos dados foi elaborado um questionário semi-estruturado, a ser preenchido de modo individual, contendo informações pessoais dos participantes - idade, sexo, categoria profissional, tempo (em anos) de trabalho efetivo na Enfermagem e área de atuação na instituição (Clínica Médica, Clínica Cirúrgica, etc.); e questões envolvendo categorias analíticas pré-estabeleci das - significado de higiene; significado de higiene corporal; aspectos a que é atribuída importância na higiene corporal pessoal e na higiene corporal do paciente; relação estabelecida entre higiene corporal e a prática de enfermagem.

Os resultados da pesquisa foram submetidos a duas vertentes de análise: a qualitativa e a quantitativa. A análise qualitativa focalizou as respostas dos participantes do estudo a duas questões: o significado de higiene e de higiene corporal; e a relação que estabeleciam entre os conceitos de higiene corporal e cuidado de enfermagem. A análise quantitativa dos dados voltou a atenção para os aspectos valorizados tanto na higiene corporal pessoal quanto na do paciente. Para a identificação desses aspectos, a resposta de cada um dos participantes foi submetida a uma leitura atentiva, considerando-se essa questão como não respondida quando o participante, ou efetivamente não a havia respondido, ou dava uma resposta de caráter geral como, por exemplo: É importante para a saúde geral. A pele sadia, íntegra, é a primeira barreira de defesa contra a infecção. Quando o participante fornecia a resposta esperada, ou seja, abordava aspectos que considerava importantes na higiene corporal pessoal e na do paciente, esses aspectos eram sublinhados e inseridos no banco de dados computadorizado. Exemplo: Manter as unhas limpas e aparadas, banho diário, escovar os dentes para evitar cárie e mau hálito, lavar os cabelos sempre para evitar seborréia.

Foram considerados todos os aspectos citados pelos participantes - higiene oral, banho, mãos, unhas (mãos e pés), cabelos, orelhas, higiene íntima, pés, narinas, barba e pêlos corporais e vestuário - discriminando-se no banco de dados os participantes que haviam citado e os que não haviam citado cada aspecto em particular. Estabelecendo-se um critério arbitrário, considerou-se os aspectos que alcançaram um percentual de citação igualou maior do que 50\% como sendo os mais valorizados, e os que alcançaram um percentual de não citação igualou maior do que $50 \%$ como sendo os menos valorizados na higiene corporal. 


\section{Resultados}

A amostra do estudo ficou limitada a 126 profissionais que devolveram o questionário devidamente preenchido até a data limite. correspondendo a 12,8\% do total geral de 986 componentes das equipes de enfermagem das instituições em que a pesquisa foi realizada. Por categoria profissional. houve uma certa semelhança na distribuição percentual dos participantes. com $26.2 \%$ de enfermeiras(os). $37,3 \%$ de técnicas(os) e $36.5 \%$ de auxiliares de enfermagem.

Houve predominância de participantes do sexo feminino (89.7\%). o que não é surpreendente, haja vista ser a Enfermagem uma profissão feminina e exercida, portanto. em sua grande maioria, por mulheres. A idade. para os participantes que a informaram (95,2\%). variou entre 19 e 66 anos, com média de 36.2 ( $\mathrm{DP}=9,1)$. Quanto ao tempo de trabalho efetivo na Enfermagem. houve o predomínio de profissionais com menos de 1 a 5 anos de (24.6\%); cumulativamente. $44,4 \%$ dos participantes tinha entre menos de 1 e 10 anos de trabalho na profissão. Finalmente, no que diz respeito à distribuição por área de atuação, verificou-se a predominância na amostra de profissionais atuando em Clínica Médica $(28,6 \%)$ e em Clínica Obstétrica $(20,6 \%)$.

A maioria dos profissionais atribuiu aos termos higiene e higiene corporal o significado de limpeza, do corpo e do meio ambiente; de um cuidado que se toma com relação a si próprio, ao meio e ao paciente; e de um cuidado que visa conforto, promove a saúde e previne doenças. Quanto à relação entre higiene corporal e cuidado de enfermagem observou-se que os respondentes consideraram haver interdependência entre os dois conceitos, entendendo a higiene corporal como uma atribuição profissional que visa ao bem-estar da clientela sob seus cuidados e como estando vinculada à imagem do profissional da Enfermagem, conforme descrito na literatura da área ${ }^{(5-8)}$

Tabela 1 - Distribuição de aspectos da higiene corporal citados ou não citados pelos profissionais de enfermagem, na higiene pessoal e na higiene do paciente. João Pessoa/PB, 2002.

\begin{tabular}{lcccccccc}
\hline \multirow{2}{*}{ Aspectos da higiene corporal } & \multicolumn{3}{c}{ Higiene pessoal (n=106) } & \multicolumn{3}{c}{ Higiene do paciente (n=88) } \\
\cline { 2 - 9 } & \multicolumn{2}{c}{ Citado } & Não citado & \multicolumn{2}{c}{ Citado } & \multicolumn{2}{c}{ Não citado } \\
\cline { 2 - 9 } & $\mathbf{f}$ & $\mathbf{9}$ & $\mathbf{f}$ & $\mathbf{9}$ & $\mathbf{f}$ & $\mathbf{\%}$ & $\mathbf{f}$ & \% \\
\hline Banho & 67 & 63,3 & 39 & 36,8 & 70 & 79,5 & 18 & 20,5 \\
Higiene oral & 62 & 58,5 & 44 & 41,5 & 54 & 61,4 & 34 & 38,6 \\
Mãos & 33 & 31,1 & 73 & 68,9 & 9 & 10,2 & 79 & 89,8 \\
Unhas (mãos e pés) & 73 & 68,9 & 33 & 31,1 & 46 & 52,3 & 42 & 47,7 \\
Cabelos & 66 & 62,3 & 40 & 37,7 & 35 & 39,8 & 53 & 60,2 \\
Orelhas & 12 & 11,3 & 94 & 88,7 & 11 & 12,5 & 77 & 87,5 \\
Higiene íntima & 20 & 18,9 & 86 & 81,1 & 12 & 13,6 & 76 & 86,4 \\
Pés & 5 & 4,7 & 101 & 95,3 & 2 & 2,3 & 86 & 97,7 \\
Narinas & 4 & 3,8 & 102 & 96,2 & 3 & 3,4 & 85 & 96,6 \\
Barba e pêlos corporais & 11 & 10,4 & 95 & 89,6 & 20 & 22,7 & 68 & 77,3 \\
Vestuário & 45 & 42,5 & 61 & 57,5 & 30 & 34,1 & 58 & 65,9 \\
\hline
\end{tabular}

$\mathrm{Na}$ Tabela 1, estão resumidos os dados referentes aos aspectos que foram citados pelos profissionais de enfermagem em suas respostas à pergunta: Cite os aspectos que você considera importantes: a) em sua higiene corporal pessoal; b) na higiene corporal do paciente. Ressalte-se que, dentre os 126 participantes do estudo, 20 não responderam o item da pergunta relacionado à higiene corporal pessoal, e 38 não responderam o item da pergunta relacionado à higiene corporal do paciente. Entre os que responderam o item da pergunta relacionado à higiene corporal pessoal $(n=106), 27$ eram enfermeiras(os), 39 eram técnicas(os) de enfermagem e 40 eram auxiliares de enfermagem. Entre os que responderam o item da pergunta relacionado à higiene corporal do paciente ( = 88), 22 eram enfermeiras(os), 34 eram técnicas(os) de enfermagem e 32 eram auxiliares de enfermagem.

Considerando-se os aspectos que alcançaram um percentual de citação igualou maior do que 50\% como sendo os mais valorizados, observa-se que, na higiene corporal pessoal, predominou a atenção a unhas $(68,9 \%)$, banho $(63,2 \%)$, cabelos $(62,3 \%)$ e higiene oral $(58,5 \%)$; e que, na higiene corporal do paciente, predominou a atenção a banho $(79,5 \%)$, higiene oral $(61,4 \%)$ e unhas $(52,3 \%)$.

Por seu turno, levando-se em consideração os aspectos que alcançaram um percentual de não citação igualou maior do que $50 \%$, coincidiram como sendo os menos valorizados, tanto na higiene corporal pessoal, quanto na higiene corporal do paciente, as mãos, as orelhas, a higiene íntima, os pés, as narinas, a barba e os pêlos corporais, e o vestuário. Especificamente na higiene corporal do paciente, o aspecto cabelo, além dos já relacionados, alcançou um percentual de não citação igual a 60,2\%, o que também o indica como um dos menos valorizados.

Através da aplicação do teste do qui-quadrado, procurou se verificar se haveria algum tipo de relação entre as variáveis idade, sexo, categoria profissional e tempo de trabalho na Enfermagem e os aspectos citados pelos participantes do estudo como sendo aqueles que consideravam importantes, tanto na higiene corporal pessoal, quanto na higiene corporal do paciente. Não foram encontrados resultados que demonstrassem haver uma associação estatisticamente significante entre as variáveis citadas e a maior ou menor valorização dos aspectos da higiene corporal, fosse a pessoal ou a do paciente.

\section{Discussão}

Para a realização deste estudo partiu-se do pressuposto de que poderia estar havendo um certo distanciamento entre o modo como os componentes da equipe de enfermagem definem os conceitos de higiene e higiene corporal e os aspectos que eles consideram como sendo importantes, tanto 
no que diz respeito à higiene corporal pessoal, como à do paciente sob seus cuidados profissionais.

$\mathrm{Na}$ higiene corporal do paciente, o banho foi o aspecto considerado mais importante pelas três categorias profissionais; a higiene oral, pelas(os) enfermeiras(os) e auxiliares de enfermagem; as unhas das mãos e dos pés, pelas(os) enfermeiras(os) e técnicas(os) de enfermagem. $\mathrm{Na}$ higiene corporal pessoal, chamou a atenção o fato de que o cuidado com as unhas e com os cabelos, mais diretamente vinculados à vaidade feminina, tenham suplantado, no número de citações dos profissionais de enfermagem, aspectos básicos da higiene corporal, como o cuidado com as mãos e com a higiene íntima.

Calcula-se que as infecções hospitalares acometem cerca de 5 a10\% dos pacientes internos, sendo as crianças e os idosos os mais susceptíveis. Entre outros fatores que fazem parte da cadeia do desenvolvimento das infecções hospitalares, ressalta-se a infecção cruzada, com a propagação de microrganismos através dos profissionais de enfermagem, dada a não observância da lavagem das mãos com a freqüência e cuidados recomendados ${ }^{(5)}$.

Recomenda-se que o profissional de enfermagem realize a lavagem das mãos e antebraços antes e após o desempenho de cuidados e/ou procedimentos simples e com baixo grau de complexidade, removendo assim a flora transitória; quando o paciente for ser submetido a procedimentos invasivos, que realize a lavagem prévia das mãos e antebraço com um tempo mínimo de sete minutos e fazendo uso de antissépticos, para remoção da flora transitória e de grande parte da flora residente ${ }^{(9)}$. Além de ser realizada antes e depois de todo contato com o paciente, é também recomendável a lavagem das mãos após o manuseio de materiais orgânicos, antes do preparo e administração de medicamentos, antes e após o uso de banheiro, bem como após qualquer contato das mãos do profissional com seu próprio corpo (cabelo, pele, nariz, orelha) ${ }^{(7)}$.

Embora seja considerado o procedimento mais importante para prevenir a infecção hospitalar, tem-se observado na prática haver pouca adesão dos profissionais de saúde à lavagem sistemática das mãos ao prestar assistência aos pacientes ${ }^{(10)}$. Esse é um problema complexo, que envolve não somente uma possível falta de motivação, como também falta de conhecimento ou outros obstáculos tais como, no caso da equipe de enfermagem: o número insuficiente de profissionais, com conseqüente sobrecarga de atividades; o tempo necessário ao cumprimento das inúmeras atribuições; a localização inadequada de lavatórios; as dermatites de contato devido ao uso de produtos químicos; entre outros possíveis.

A despeito dos obstáculos apontados, considera-se válido destacar que o percentual de respondentes que citaram as unhas das mãos e dos pés $(68,9 \%)$ como sendo um aspecto que consideram importante na higiene corporal pessoal é exatamente igual ao percentual de respondentes que não citaram as mãos $(68,9 \%)$ como um desses aspectos. Que possíveis explicações podem ser apresentadas para o fato? Será que, por se tratar de uma profissão predominantemente feminina, o cuidado higiênico com as unhas, diretamente vinculado à vaidade e à aparência, está sendo levado mais em consideração pelos profissionais de enfermagem do que o cuidado higiênico das mãos, com as quais grande parte do cuidado de enfermagem é realizado?

De modo geral, observou-se que o cuidado com as orelhas não foi um aspecto considerado importante pelos profissionais de enfermagem que participaram deste estudo, seja na higiene corporal pessoal ou na higiene corporal do paciente. Ao não se atribuir a devida importância à higiene das orelhas, desconsidera-se a possibilidade de que as otites ou as infecções secundárias locais possam ser fruto do uso do estetoscópio sem limpeza prévia das ogivas; ou de que, ao se coçar ou tocar as orelhas sem a lavagem posterior das mãos, há o risco de se estar carreando microrganismos da polpa digital dos dedos para a orelha, ou vice-versa. Enquanto que no primeiro exemplo é a integridade auricular do profissional que está em risco, no segundo exemplo tanto há o risco de contaminação do profissional, quanto do paciente sob seus cuidados.

Em se tratando de uma profissão em que predominam pessoas do sexo feminino, chama a atenção o fato de que $81,1 \%$ dos profissionais não tenham citado a higiene íntima como um aspecto que consideram importante na higiene corporal pessoal. Do ponto de vista da higiene corporal do paciente, seja ele do sexo masculino ou do sexo feminino, é preocupante verificar que $86,4 \%$ dos profissionais de enfermagem não tenham citado esse aspecto como sendo um dos que consideram importante, o que pode estar expondo a clientela sob seus cuidados a risco de infecções e a desconfortos desnecessários.

Conforme pode ser visto na Tabela 1, o cuidado com os pés e o cuidado com as narinas não foram considerados importantes pelos profissionais de enfermagem que participaram deste estudo, nem na higiene corporal pessoal, nem na higiene corporal do paciente. A não atribuição de importância a esses aspectos da higiene corporal se distribuiu de forma semelhante entre as três categorias profissionais. Finalmente, a depender das respostas obtidas neste estudo, barba e pêlos corporais também não são aspectos considerados pelos profissionais de enfermagem como sendo importantes, seja na higiene corporal pessoal ou na higiene corporal do paciente.

\section{Considerações Finais}

De modo geral, podemos concluir que a preocupação dos profissionais de enfermagem que participaram deste estudo parece estar focada em partes visíveis do corpo, seguindo uma perspectiva de moralidade, de decência e boa apresentação, a exemplo do que Vigarello(2) discute em sua obra. Esses resultados são preocupantes e apontam para a possibilidade de que os profissionais de enfermagem estejam veiculando, por falta de cuidados higiênicos básicos, microorganismos causadores de infecções diretas ou cruzadas nos pacientes, preocupação constante das Comissões de Controle de Infecção Hospitalar. Além disso, fazem com que questionemos como os aspectos relacionados à higiene corporal foram transmitidos a essas pessoas durante o processo de socialização primária; e como essa temática está sendo abordada durante a formação profissional e durante a Educação Continuada.

Quando perguntados acerca da relação que estabeleciam entre higiene corporal e cuidado de enfermagem, esses profissionais entenderam os dois conceitos como sendo interdependentes e destacaram a higiene corporal como um aspecto importante para a imagem do profissional e como um cuidado básico para o alcance da meta de proporcionar conforto e bem-estar ao paciente. Os resultados obtidos se contrapõem à visão expressa por eles acerca da higiene corporal como um cuidado fundamental, que proporciona bem-estar e conforto e que promove a saúde e previne doenças.

A principal conclusão a que se chega ao analisar os dados deste estudo é que há, aparentemente, pouca coerência entre o pensar e o agir dos profissionais de enfermagem no que diz respeito aos conceitos de higiene e higiene corporal. Confirmamos, pois, o pressuposto do distanciamento entre o modo como os componentes da equipe de enfermagem definem os conceitos de higiene e higiene corporal e os aspectos que eles consideram como sendo importantes, tanto no que diz respeito à higiene corporal pessoal, como à do paciente sob seus cuidados profissionais.

Os problemas identificados devem resultar em alguns 
desdobramentos para o ensino, para a assistência e para o gerenciamento das atividades de enfermagem. Acreditamos na necessidade de modificar a imagem que emergiu dos resultados deste trabalho, ou seja, na necessidade de provocar uma alteração nas regras (hábitos e costumes) dos profissionais de enfermagem no que diz respeito à higiene corporal pessoal e à higiene corporal do paciente.

Durante a formação profissional das três categorias -enfermeiras(os), técnicas(os) e auxiliares de enfermagem os aspectos abordados neste estudo reclamam uma atenção maior. Durante o desempenho das atividades profissionais, há a possibilidade de reverter a situação por meio de ações efetivas e regulares de Educação Continuada. Essa é uma atividade que as(os) gerentes de enfermagem das instituições prestadoras de serviços de internação hospitalar não podem (ou não devem) subestimar.

Finalmente, do ponto de vista da administração e da estrutura dos serviços de saúde, recomendamos uma maior atenção às instalações dos vestiários para os profissionais de enfermagem, de preferência tendo banheiros aparelhados com chuveiros e com duchas para higiene íntima, de modo a estimular a alteração nas regras (hábitos e costumes) dos profissionais de enfermagem no que diz respeito à higiene corporal pessoal.

\section{Referências}

1. Kloetzel K. Temas de saúde: higiene física e do ambiente. São Paulo: EPU; 1980. 312 p. ii.
2. Vigarello G. O limpo e o sujo: uma história da higiene corporal. São Paulo: Martins Fontes; 1996. 297 p.

3. Nightingale F. Notas sobre enfermagem: o que é e o que não é. São Paulo: Cortez; 1989. 174 p.

4. Ministério da Saúde (BR), Conselho Nacional de Saúde. Resolução nO 196, de 10 de outubro de 1996: diretrizes e normas regulamentadoras de pesquisas envolvendo seres humanos. Brasília (DF); 199.24 p.

5. Atkinson LD, Murray ME. Fundamentos de enfermagem: introdução ao processo de enfermagem. Rio de Janeiro: Guanabara: 1989.618 p. il.

6. Ellis JR, Nowlis EA. Nursing: a human needs approach. 58 ed. Philadelphia: Lippincott; 1994.

7. Potter PA, Perry AG. Grande tratado de enfermagem: prática clínica e prática hospitalar. $3^{8}$ ed. São Paulo: Santos; 2001.999 p. ii.

8. Smeltzer SC, Bare BG. Brunner/Suddarth: tratado de enfermagem médico-cirúrgica. $7^{8}$ ed. Rio de Janeiro: Guanabara Koogan; 1994.2 v. v. 1.

9. Graziano KU. Fatores de risco relacionado a todo paciente cirúrgico: anti-sepsia, degermação e preparo pré-operatório da pele. In: Lacerda RA, coordenadora. Buscando compreender a infecção hospitalar no paciente cirúrgico. São Paulo: Atheneu; 1992. 177 p. il. p.52-9.

10. Trabasso P. Adesão à lavagem das mãos ainda é pequena. Revista Meio de Cultura: Eurofarma, São Paulo 2002;5(19):15-

Data de Recebimento: 20/08/2003

Data de Aprovação: 22/12/2003 